\title{
Vibrational Characterization of Synthetic Eumelanin by Means of Raman and Surface Enhanced Raman Scattering
}

\author{
Giuseppe Perna, Maria Lasalvia, Crescenzio Gallo, Giuseppe Quartucci and Vito Capozzi*
}

Dipartimento di Medicina Clinica e Sperimentale, Università di Foggia, Viale Pinto, 71122 - Foggia, Italy

\begin{abstract}
Eumelanin biopolymer synthetized from L-DOPA has been characterized by Raman and Surface Enhanced Raman Spectroscopy (SERS). Although Raman spectra of eumelanin were scarcely resolved, SERS measurements of this biopolymer on nanostructured gold substrate, provided a large enhancement of vibrational peaks related to functional groups adherent to the substrate, so that well resolved vibrational spectra were obtained. The SERS peaks correspond to vibrational modes related to the monomeric units of eumelanin. So, SERS spectra can be used to identify the monomeric content of eumelanin and resolve the presence of vibrational modes which have been calculated and predicted according to different theoretical models and previously observed in vibrational spectra of single monomeric units.
\end{abstract}

Keywords: Eumelanin, Raman, SERS, vibrational modes.

\section{INTRODUCTION}

Eumelanin is an important natural pigment, which is produced in epidermal melanocytes by tyrosinase-catalized oxidation of tyrosine [1]. Its main biological function is a protection role against the biochemical damages induced by solar exposure, particularly by UV radiation, which is strongly absorbed and converted into heat through nonradiative processes [2]. Such pigment, besides its important biological role, has a few physical properties, like broad UV and visible absorption spectra [3-5], electrical conductivity [6] and photoconductivity [7], which are interesting for a possible integration in photovoltaic [8] and electronic devices [9].

The physical properties are strongly dependent on the structural organization of the biopolymer. It is fairly accepted that the basic molecular units (monomers) of eumelanin are 5,6-dihydroxyindole (DHI), 5.6-dihydroxyndole-2-carboxylic acid (DHICA) and their redox forms 5,6-indolequinone (IQ) and semiquinone (SQ) [2]. These units consist of one aromatic and one pyrrole ring, which are fused to form an indolic structure, as shown in Fig. (1). Pyrrole di- and tri-carboxylic acids can also be present inside the biopolymer as a result of oxidation of DHI and DHICA units [10]. Although several recent studies have supported a structural model based on the aggregation of few stacked planar sheets of the above oligomeric structures to form eumelanin protomolecules [5, 11], signals from single functional groups inside the eumelanin compound are scarcely resolved in literature. In fact, such studies are mainly based on the explanation of optical properties of eumelanin biopolymers, as absorption [12] and fluorescence spectra [13, 14]. The absorption spectrum at room temperature of the synthetic eumelanin solution is

*Address correspondence to this author at the Dipartimento di Medicina Clinica e Sperimentale, Università degli studi di Foggia, Viale Pinto, I71122 Foggia, Italy; Tel: +39 0881588053; E-mail: vito.capozzi@unifg.it characterized by a strong absorption in the UV and visible spectral range, with a nearly featureless lineshape and absorbance values monotonically increasing from near-IR to UV spectral region [2, 3, 12, 13]. Fluorescence spectrum of eumelanin is also characterized by broad emission bands, due to the contribution of many chemically distinct macromolecules [2, 3, 13, 14]. Such optical spectra have been explained as due to the overlapping contribution of many eumelanin monomers whose energy is close to each other. a)<smiles>Oc1cc2cc[nH]c2cc1O</smiles>
$\mathrm{DHI}$

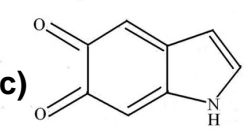
IQ<smiles>O=C(O)c1cc[nH]c1C(=O)O</smiles>
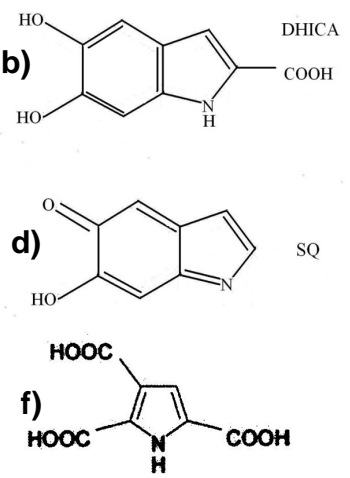

Fig. (1). Schematic representation of the basic monomeric units of eumelanin: a) DHI, b) DHICA, c) IQ, d) SQ [18] and products of oxidation of DHI and DHICA units: e) pyrrole-2,3-dicarboxylic acid, f) pyrrole-2,3,5-tricarboxylic acid [10].

Raman spectroscopy can be a useful method to identify the molecular groups of the monomeric units that aggregate themselves to form an eumelanin macromolecule, because each monomer has proper spectral features. Normal Raman spectra of eumelanin are usually characterized by a low signal-to-noise ratio, because of the low exciting power required to minimize thermal and photochemical degradation of the sample. In fact, the Raman technique has been applied to investigate, both theoretically $[15,16]$ and experimentally 
[10, 17-20], the vibrational finger-print of eumelanin and eumelanin precursors. In particular, simulated Raman spectra of eumelanin components, as DHI, IQ, SQ [15] and DHICA [16], are very rich of features related to different vibrational modes of functional groups inside such components. Nonetheless, vibrational structures are scarcely resolved in experimental Raman spectra because broadening and overlapping effects occur among Raman features centred at wavenumber values very close to each other [10, 17-19]. On the contrary, Surface Enhanced Raman Scattering (SERS) can be an appropriate tool for the vibrational characterization of eumelanin. Indeed, such technique yields a strong increase of the Raman signal for those functional groups adsorbed on or located very close to a rough nanostructured metal surface, as a result of $i$ ) a resonance effect of both exciting laser field and scattered Raman field with the surface plasmons of metal nanostructure (electromagnetic field enhancement) and ii) the increased Raman cross-section of the functional group attached to the metal nanostructure (chemical enhancement) [21-23]. Therefore, SERS spectra are characterized by a higher sensitivity at low excitation laser intensity and a better resolution with respect to normal Raman spectra.

Despite of the advantages of the SERS technique, a main drawback is related to the difficulties in fabricating a reliable, high sensitive and reproducible (uniformly roughened) nanoscale substrate, onto which the biopolymer can be deposited and from which repeatable SERS signal can be collected. Among the commercially available SERS substrates, a suitable sample for signal reproducibility and enhancement is a gold nanostructured one known as "Klarite" substrate [24, 25]. In fact, manufacturer tests report relative standard deviations in signal levels of $<10 \%$ with laser excitation at 633 or $785 \mathrm{~nm}$ [26], although information about SERS signal reproducibility with Klarite substrates are scarcely presented in the literature. Nonetheless, the reproducibility of the SERS spectrum of a sample including many functional groups (i.e. many vibrational modes are possible) depends not only on the uniformity of substrate morphology but also on the orientation of the functional groups on the substrate. Instead, the enhancement of Raman signal on Klarite substrate is reported as varying from a factor of about 100 [27] to an extremely high factor, so that vibrational signal which are very weak in Raman spectra become well resolved in SERS spectra [28, 29]. The plasmon absorbance bands of Klarite substrate are located at $577 \mathrm{~nm}$ and $749 \mathrm{~nm}$. Consequently, such substrate is SERS active only when excited from laser lines close to such bands. Moreover, such substrates are designed to be used, once opened, just prior the measurements to avoid any possible surface contamination.

To our knowledge, very few works about SERS spectra of eumelanin have been published up to now: in fact, Centeno et al. investigated SERS spectra of Sepia eumelanin [10] and Martin-Sanchez et al. measured SERS spectra of fungal eumelanin [30]. However, these works do not report any information neither about the enhancement factor of Raman signal nor the reproducibility of spectra. In addition, both such types of eumelanin are obtained from biological samples. An important issue to remark is that eumelanin in biological organisms is firmly bound to proteinaceous components, through covalent or ionic bonds [31]. It is important to remove the protein component in order to study the physical properties of the eumelanin biopolymer. A method to prepare eumelanin samples without the protein component is to synthesize it non-enzymatically, for example through oxidation of tyrosine with hydrogen peroxide or through oxidation and polymerization processes induced in L-DOPA (3,4- dihydroxy-L-phenylalanine) aqueous solution. The sample obtained in such a procedure is known as synthetic eumelanin: it can be considered a good model to investigate the properties of eumelanin biopolymer independently of the protein coat. SERS spectra of synthetic eumelanin have not been investigated up to now, although they are interesting because they could resolve single vibrational modes related to functional groups present in monomeric units and, consequently, they could yield information about the chemical composition of the main eumelanin basic units and their presence inside the biopolymer. For this aim, a comparison of the experimental SERS spectra with the simulated and experimental Raman spectra of the monomeric units is essential.

In this work, we report on Raman and SERS spectra of synthetic eumelanin biopolymer. Raman spectra typically consists of two broad bands, centred at about $1400 \mathrm{~cm}^{-1}$ and $1590 \mathrm{~cm}^{-1}$, which are due to the convolution of several Raman-active vibrational modes within the indolic structure of eumelanin. An enhancement of the Raman intensity and of signal-to-noise ratio is observed for SERS measurements. Therefore, the SERS spectra are better resolved with respect to the Raman ones. The spectral position of the features observed in SERS spectra are consistent with those reported in literature for similar compounds, based on indole and pyrrole structure, as well as with the calculated spectra of the main eumelanin components. The identification and attribution of such spectral features can contribute to the determination of the chemical composition and structural organization of the biopolymer.

\section{MATERIALS AND METHODS}

Samples of synthetic eumelanin were prepared starting from aqueous solution of $5 \mathrm{mM}$ commercial L-DOPA (Sigma-Aldrich) in $5 \mathrm{mM} \mathrm{Na-phosphate} \mathrm{buffer} \mathrm{(pH} \mathrm{7.0).} \mathrm{L-}$ DOPA solutions were aerated and stirred at room temperature for approximately $72 \mathrm{~h}$, till they became darkbrown, due to spontaneous oxidation and polymerization $[32,33]$.

The sample for Raman investigation was obtained by depositing a droplet of eumelanin solution onto a microscopy glass slide, previously rinsed with purified water. The droplet was left in air at room temperature until the liquid was completely evaporated: a dark-brown deposit was left onto the glass slide, as shown in Fig. (2a).

The sample for SERS measurements was obtained by deposition and evaporation of a droplet of eumelanin solution onto a commercially available Klarite SERS substrate (currently manufactured by Renishaw Diagnostic). Such substrate consists of gold coated nanostructured silicon holes arranged according to a square lattice framework [24, 25]. An optical image of the SERS substrate before and after droplet deposition and solvent evaporation (i.e. just before SERS measurements) is shown in Fig. (2b, c), respectively. 

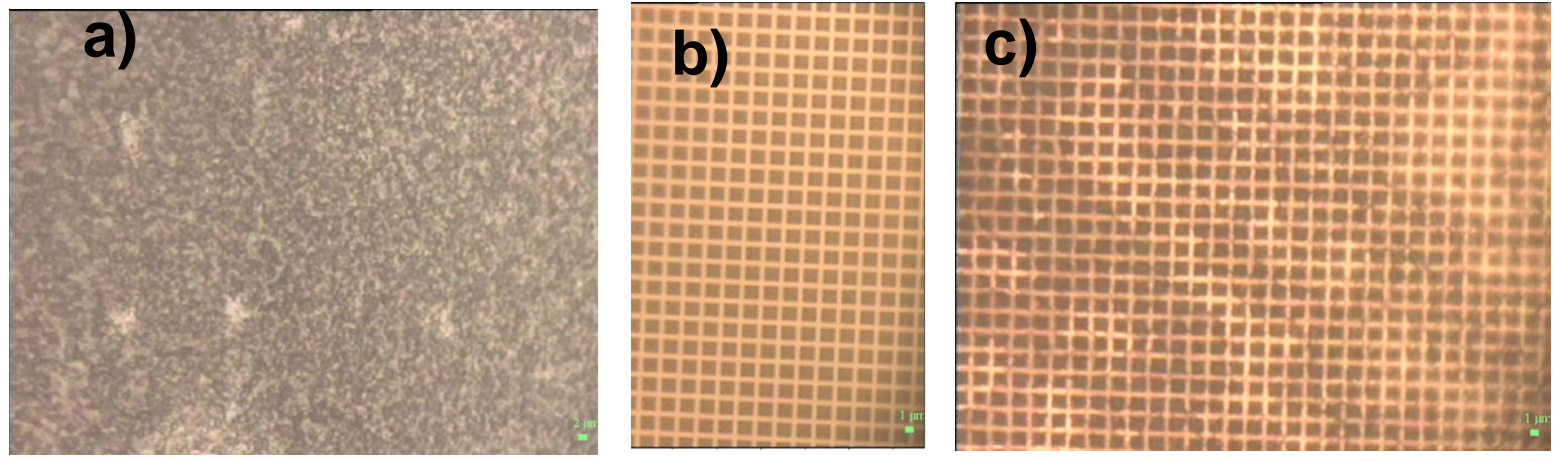

Fig. (2). Optical images of (a) an air-dried droplet of synthetic eumelanin solution deposited onto a microscopy glass slide, (b) a Klarite SERS substrate and (c) an air-dried droplet of synthetic eumelanin solution deposited onto the Klarite SERS substrate.

It is clearly visible that synthetic eumelanin considerably wets the substrate.

The Raman measurements were carried out at room temperature by means of a Raman confocal microspectrometer using the $633 \mathrm{~nm}$ line of $\mathrm{He}-\mathrm{Ne}$ and the $488 \mathrm{~nm}$ line of Ar-ion as laser sources and a notch filter $\left(200 \mathrm{~cm}^{-1}\right.$ line-with) to suppress the laser scattered light. SERS measurements were carried out using the $633 \mathrm{~nm}$ excitation line, because such wavelength is closer to a plasmon absorbance wavelength of the Klarite substrate with respect to the $488 \mathrm{~nm}$ line. The Raman signal was analysed by means of a holographic grating with 1800 grooves $/ \mathrm{mm}$ and it was detected by means of a cooled CCD (at $223 \mathrm{~K}$ ). The laser beam was focused, by an Olympus optical microscope with a $\times 100$ objective, on the investigated sample, obtaining an illuminated spot of few $\mu \mathrm{m}$ diameter. The laser power at the sample surface was about $0.04 \mathrm{~mW}$ and the integration time of each spectrum was $30 \mathrm{~s}$. The mean spectral resolution was $4 \mathrm{~cm}^{-1}$. In particular, 10 Raman spectra (for each excitation line) and 12 SERS spectra have been measured, by focusing the laser spot onto different points of the sample. Then, the contribution of the fluorescence emission has been subtracted to each spectrum, using a second order polynomial function. After such pre-treatment procedure, the Raman and SERS spectra were averaged, in order to obtain a mean Raman and SERS spectrum.

The line shape of the mean Raman spectrum has been analysed by a fitting procedure to the sum of several Raman bands represented by Gaussian functions. Such line shape analysis has been carried out after calculation of the second derivative of the experimental data in order to locate the spectral position of the Raman peaks according to second derivative minima. In such a way, the fitting parameters can be reduced to the area and the spectral linewidth of each Gaussian function. Second derivative method has also been used to locate the spectral position of SERS peaks, although a fitting analysis has not been performed in such a case because a large number of features involves too many fitting parameters, which could affect with a large degree of uncertainties the fitting procedure till to invalidate it.

\section{RESULTS AND DISCUSSION}

\section{Raman Spectra}

Typical raw Raman spectra collected from an air dried drop of synthetic eumelanin at room temperature with two different laser wavelengths, a) 633 and b) $488 \mathrm{~nm}$, are shown in Fig. (3). Such spectra include both Raman and fluorescence signals. In particular, the broad bands at about $1590 \mathrm{~cm}^{-1}$ and $1400 \mathrm{~cm}^{-1}$ refer to Raman signal, because their spectral position does not change with the excitation wavelength, whereas the background signal is related to the fluorescence contribute. The intensity of Raman signal appears to be larger in the spectrum b) excited with $488 \mathrm{~nm}$ line because the intensity of Raman signal scales with a $\propto 1 / \lambda^{4}$ dependence on the excitation wavelength $\lambda$. Such behaviour is in agreement with that shown in [17] for Sepia eumelanin samples.

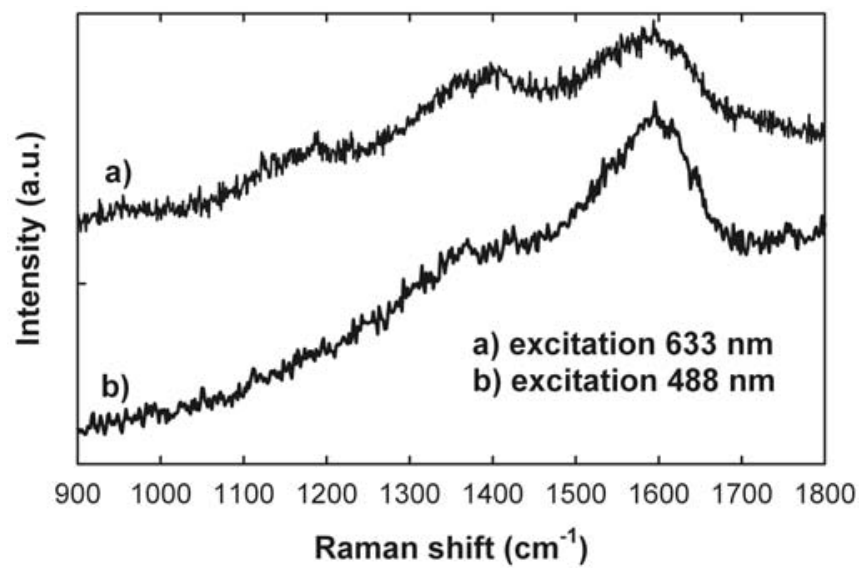

Fig. (3). Typical Raman spectra of an air-dried synthetic eumelanin droplet deposited on a glass substrate, excited by a) the $633 \mathrm{~nm}$ and b) the $488 \mathrm{~nm}$ laser line. The spectra have been intensity shifted for clarity purpose.

In the following, we have considered only Raman spectra measured with the $633 \mathrm{~nm}$ excitation line, because of the lower absorption of such wavelength radiation with respect to the $488 \mathrm{~nm}$ one. Consequently, a better minimization of thermal and photochemical degradation of the sample. The average Raman signal of the background-subtracted spectra collected from a synthetic eumelanin sample at room temperature is shown in Fig. (4) (continuous line). The low level of standard deviation values (dotted line) points out that the Raman signal is quite reproducible among the different sites where it has been collected. This behaviour implies a homogeneous composition of the biopolymer samples. In fact, the spectrum in Fig. (4) is dominated by the two strong and broad bands centred at about 1400 and 1590 $\mathrm{cm}^{-1}$, with other weaker intensity bands appearing at lower wavenumbers. Such bands are related to Raman active 
vibrational modes involving different atoms, as carbon, oxygen, hydrogen and nitrogen, which are the main constituent of eumelanin. These atoms are organized according to an indolic structure, with several functional groups bonded to it, mainly hydroxyl groups $\mathrm{OH}$, carbonyl groups $\mathrm{C}=\mathrm{O}$, carboxylic acid $\mathrm{COOH}$ and $\mathrm{NH}$ groups. The bonds of such functional groups to the indolic structure yields the formation of the different basic monomeric units of eumelanin. Therefore, it results quite difficult to assign precisely the observed Raman features of eumelanin, because of the large amount of different vibrational modes involving the many functional groups inside the biopolymer. The overall shape of the Raman spectrum is similar to those reported for synthetic eumelanin sample [19, 34] as well as that simulated by Sangaletti et al. [19], based on the calculations of Powell et al. [15] about the vibrational modes of eumelanin monomers DHI, IQ and SQ, so confirming the presence of such units inside the analysed sample.

To better analyze the functional groups characteristic of the eumelanin structure and to point out some spectral features which may be hidden in the experimental spectra, the typical Raman spectrum (Fig. 4) has been analysed by means of Gaussian functions deconvolution. The results of this fitting procedure are shown as red dashed lines in Fig. (5) and the spectral position of Raman peaks are listed in Table 1. It is clearly evident that the sum of Gaussian functions (red continuous line) is well fitted to the experimental spectrum (black continuous line).

Table 1. Spectral Position of Raman Peaks

\begin{tabular}{|c|c|c|}
\hline Raman Peaks $\left(\mathrm{cm}^{-1}\right)$ & SERS Peaks $\left(\mathrm{cm}^{-1}\right)$ & Assignment \\
\hline & 927 & $\begin{array}{l}\mathrm{O}-\mathrm{H} \text { out of plane deformation; } \\
\mathrm{C}-\mathrm{H} \text { out of plane deformation }\end{array}$ \\
\hline \multirow[t]{3}{*}{942} & & $\begin{array}{l}\mathrm{O}-\mathrm{H} \text { out of plane deformation; } \\
\mathrm{C}-\mathrm{H} \text { out of plane deformation }\end{array}$ \\
\hline & 1027 & $\mathrm{C}-\mathrm{H}$ in plane deformation \\
\hline & 1075 & $\mathrm{C}-\mathrm{H}$ in plane deformation \\
\hline \multirow[t]{2}{*}{1078} & & $\mathrm{C}-\mathrm{H}$ in plane deformation \\
\hline & 1123 & $\mathrm{~N}-\mathrm{H}$ in plane deformation \\
\hline \multirow[t]{2}{*}{1139} & & $\mathrm{~N}-\mathrm{H}$ in plane deformation \\
\hline & 1170 & $\mathrm{C}-\mathrm{H}$ in plane deformation \\
\hline \multirow[t]{3}{*}{1200} & & $\mathrm{C}-\mathrm{H}$ in plane deformation \\
\hline & 1213 & $\mathrm{C}-\mathrm{H}$ in plane deformation \\
\hline & 1258 & $\mathrm{C}-\mathrm{O}$ stretching in $\mathrm{COOH}, \mathrm{C}-\mathrm{C}$ and $\mathrm{C}-\mathrm{N}$ stretching in pyrrole \\
\hline \multirow[t]{2}{*}{1268} & & $\mathrm{C}-\mathrm{O}$ stretching; combination of $\mathrm{C}-\mathrm{O}$ stretching and $\mathrm{O}-\mathrm{H}$ deformation in carboxylic acid \\
\hline & 1303 & $\mathrm{O}-\mathrm{H}$ deformation in carboxylic acid \\
\hline \multirow[t]{3}{*}{1335} & & $\mathrm{C}-\mathrm{N}$ stretching in pyrrole \\
\hline & 1345 & $\mathrm{C}-\mathrm{N}$ stretching, indole ring vibration \\
\hline & 1389 & $\mathrm{C}=\mathrm{C}, \mathrm{C}=\mathrm{N}$ in plane vibration in pyrrole \\
\hline \multirow[t]{4}{*}{1407} & & $\mathrm{C}=\mathrm{C}, \mathrm{C}=\mathrm{N}$ in plane vibration in pyrrole \\
\hline & 1422 & $\mathrm{C}=\mathrm{C}, \mathrm{C}=\mathrm{N}$ in plane vibration in pyrrole \\
\hline & 1469 & $\mathrm{C}=\mathrm{C}, \mathrm{C}=\mathrm{N}$ in plane vibration in pyrrole \\
\hline & 1510 & Indole ring vibration, $\mathrm{C}=\mathrm{C}$ in plane vibration in pyrrole \\
\hline \multirow[t]{3}{*}{1528} & & $\mathrm{C}=\mathrm{C}$ in plane vibration in pyrrole \\
\hline & 1545 & Indole ring vibration \\
\hline & 1587 & Indole ring vibration \\
\hline \multirow[t]{4}{*}{1617} & & Indole ring vibration \\
\hline & 1621 & Indole ring vibration \\
\hline & 1663 & $\mathrm{C}=\mathrm{O}$ stretching in quinone \\
\hline & 1703 & $\mathrm{C}=\mathrm{O}$ stretching in $\mathrm{COOH}$ \\
\hline \multirow[t]{2}{*}{1717} & & $\mathrm{C}=\mathrm{O}$ stretching in quinone and $\mathrm{COOH}$ \\
\hline & 1735 & $\mathrm{C}=\mathrm{O}$ stretching in $\mathrm{COOH}$ \\
\hline \multirow[t]{2}{*}{1771} & & $\mathrm{C}=\mathrm{O}$ stretching in $\mathrm{COOH}$ \\
\hline & 1775 & $\mathrm{C}=\mathrm{O}$ stretching in $\mathrm{COOH}$ \\
\hline
\end{tabular}




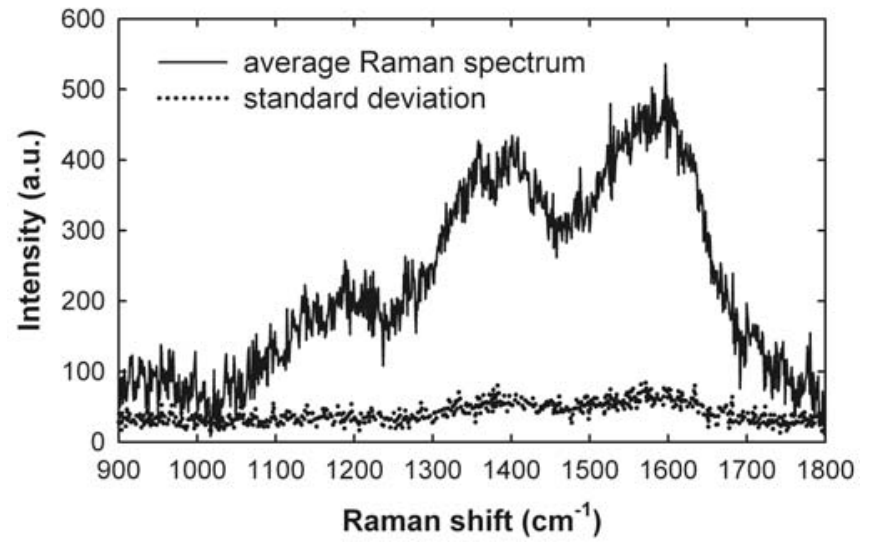

Fig. (4). Average Raman spectrum (continuous line) and relative standard deviation (dotted line) of an air-dried synthetic eumelanin droplet deposited on a glass substrate and excited by the $633 \mathrm{~nm}$ line. The spectrum has been averaged among 10 spectra collected from different points of the sample.

A possible assignment of Raman bands can be based on several works related to vibrational properties of synthetic $[19,34]$ and natural [10, 17, 18] eumelanins and other aromatic compounds containing indole and pyrrole groups [20, 35-40]. From the analysis of Fig. (5), the broad band at about $1590 \mathrm{~cm}^{-1}$ results from the contribution of four Raman bands centred at $1771,1717,1617$ and $1528 \mathrm{~cm}^{-1}$. The very weak feature at $1771 \mathrm{~cm}^{-1}$ in Fig. (5) corresponds to a spectral position characteristic of $\mathrm{C}=\mathrm{O}$ stretching vibrational mode of carboxylic acid: it can be due to carboxylic derivatives of pyrrole (originating from oxydation of DHI and DHICA units) [10]. The weak band at $1717 \mathrm{~cm}^{-1}$ in Fig. (5) can be assigned to the $\mathrm{C}=\mathrm{O}$ stretching mode, which is present in the quinone structure and in the carboxylic acid group [37]. The band centred at $1617 \mathrm{~cm}^{-1}$ can be attributed to $\mathrm{C}=\mathrm{C}$ aromatic ring vibrations of the indole structure [37]. Further, the band at $1528 \mathrm{~cm}^{-1}$ can be assigned to $\mathrm{C}=\mathrm{C}$ and $\mathrm{C}=\mathrm{N}$ ring stretching modes of the pyrrole structure [34].

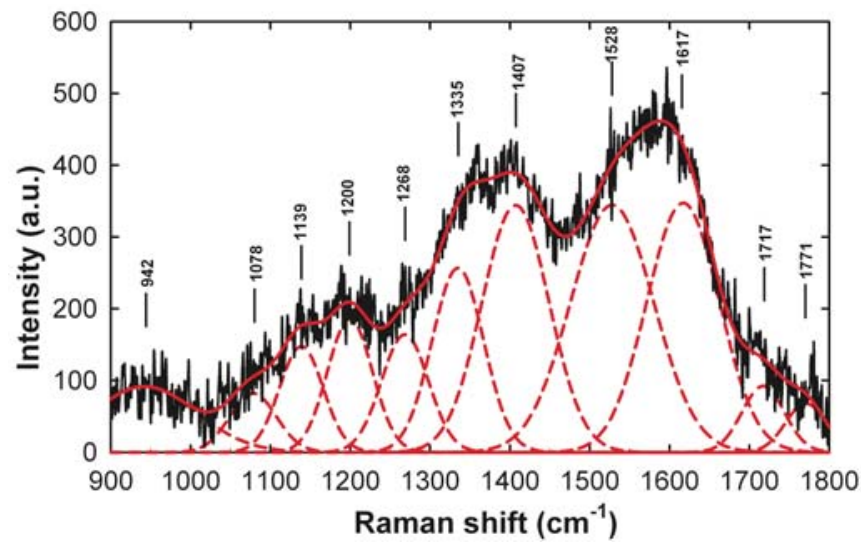

Fig. (5). Fitting analysis of the experimental average Raman spectrum (black continuous line) obtained by means of Gaussian functions (red dashed lines): the sum of the Gaussian bands (red continuous line) is in good agreement with the experimental spectrum. The quality of the fit is remarkable: in fact, the $r^{2}$ values is 0.99927 .

The broad band at $1400 \mathrm{~cm}^{-1}$ results from the overlapping of several vibrational modes, mainly: $i$ ) the $\mathrm{C}=\mathrm{C}$ and $\mathrm{C}=\mathrm{N}$ ring stretching mode of the pyrrole structure described above, which are responsible of the band at $1407 \mathrm{~cm}^{-1}$ in Fig. (5); ii) the C-N stretching mode of the pyrrole structure [10], which may contribute to the band centred at $1335 \mathrm{~cm}^{-1}$ in Fig. (5); iii) the $\mathrm{C}-\mathrm{O}$ stretching mode and combination bands due to $\mathrm{C}-\mathrm{O}$ stretching and $\mathrm{O}-\mathrm{H}$ deformation of the carboxylic acid [38-40]: such modes can be responsible of the Raman band at $1268 \mathrm{~cm}^{-1}$ in Fig. (5).

The lower intensity bands at about 1200, 1139, 1078 and $942 \mathrm{~cm}^{-1}$ in Fig. (5) are related to deformation modes. In particular, the bands at 1200 and $1078 \mathrm{~cm}^{-1}$ are probably due to $\mathrm{CH}$ in-plane deformation modes [34, 37]; the band at 1139 $\mathrm{cm}^{-1}$ can be related to $\mathrm{NH}$ in-plane deformation mode of the pyrrole structure [34], whereas the band at $942 \mathrm{~cm}^{-1}$ can be related to $\mathrm{CH}$ out-of-plane deformation mode [34] and to the $\mathrm{OH}$ out-of-plane deformation mode [39].

Despite of the previous attributions are widespread in the eumelanin literature, the normal Raman spectra do not provide information about the eumelanin components with large details. In fact, as predicted by Powell [15], some basic eumelanin units, i.e. DHI, SQ and IQ, present Raman spectra characterized by a lot of peaks when they appear in gaseousphase monomers: each peak has a proper intensity value, according to the Raman scattering activity of the corresponding vibrational mode. So, by considering broadening and shifting effects due to the formation of macromolecules and polymerization process, single Raman peaks related to a corresponding vibrational mode can be hardly resolved in the Raman spectra of eumelanin samples consisting of air dried deposit, film and powders. The identification of vibrational modes contributing to the Raman spectrum cannot be improved by increasing the number of Gaussian functions during the deconvolution procedure, because of the consequent increase of freedom degrees. Therefore, we retain that each band resulting from the deconvolution procedure might include, in addition to the vibrational modes described above, also the overlapped and unresolved contribution of other different vibrational modes of eumelanin components.

\section{SERS Spectra}

Differently from the Raman technique, SERS spectroscopy can provide spectra characterized by well resolved features because of the enhancement of Raman scattering by the molecules adsorbed on nano-structured metal surfaces. This enhancement effect is visible in the average SERS spectrum of synthetic eumelanin sample, shown in Fig. (6). Indeed, the intensity of the average SERS spectrum is about 5-times larger than that of the average Raman spectrum (Fig. 4). This low value of enhancement factor with respect to those reported in literature for SERS effect can be related to the thickness of the eumelanin sample and the partial self-absorption of light scattered from the layer adsorbed on the metal surface by the overlaying layers. Although the single SERS spectra show a good reproducibility, the values of standard deviation (dotted line) of the average SERS spectrum are larger than the corresponding values of the Raman spectrum. As SERS enhancement involves only vibrational modes characteristic of functional groups which are located very close to the nanostructured substrate, the variations observed among spectra may be related to different molecular orientation of 
the functional groups of eumelanin components on the SERS substrate surface and, consequently, to different molecular interactions with metal surface. We remark that the Raman signal of nanostructured substrate does not influence the SERS spectrum. In fact, the Raman signal of the substrate is characterized by a weak intensity and featureless spectrum (data not shown). The SERS spectrum shown in Fig. (6) is characterized by well resolved spectral features, corresponding to vibrational modes characteristic of functional groups inside the eumelanin structure. So, the nanostructured surface does not promote the preferential orientation of any functional groups, which are randomly oriented during the deposition process. This matter is demonstrated by the overall shape of SERS spectrum in Fig. (6): it results quite similar to that of Raman spectrum in Fig. (4) (apart the presence of more resolved features and enhanced signal in the former). This shape is caused by the cross-section of Raman effect of each functional group composing the eumelanin biopolymer. If there were a preferential orientation of a specific functional groups on the substrate, the signal from this group would be largely enhanced with respect to that from the other groups and a very strong and resolved peak should be present in Fig. (6).

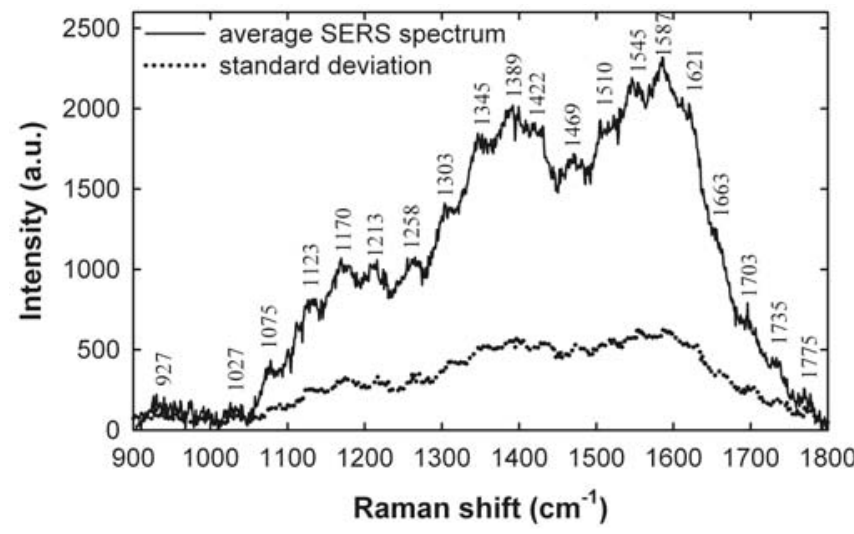

Fig. (6). Average SERS spectrum (continuous lines) of an air-dried synthetic eumelanin droplet deposited on a commercial Klarite SERS substrate excited by the $633 \mathrm{~nm}$ line. The spectrum has been averaged among 12 spectra collected from different points of the sample. The spatial variability of SERS spectra, characterized by the standard deviation spectrum (dotted line) occurs because of different molecular interactions with metal surface.

The SERS spectrum in Fig. (6) is fairly similar to that obtained by Centeno et al. [10] for Sepia eumelanin, whereas it is rather different from that measured by Martin-Sanchez et al. [30] for various types of fungal eumelanin. Such differences are probably related to the different synthesis pathways of fungal eumelanin with respect to the Sepia and synthetic ones [30] and, consequently, to the different residual components of the synthesis processes.

The wavenumber of the spectral features resolved in Fig. (6) are summarized in Table $\mathbf{1}$. The assignment of the SERS peaks cannot be achieved with certainty, because wavenumber shifts may occur in the SERS spectra with respect to the Raman ones for those vibrational modes involving atoms bonded to the substrate surface [23]. Moreover, a SERS spectrum can show some deviations in the relative intensities of the spectral features compared with the normal Raman spectrum of the same sample, as a consequence of different enhancement mechanisms for different Raman modes [23]. Most of the SERS peaks can be attributed by means of comparison with literature data of experimental $[10,16,30,41]$ and theoretical $[15,35,36]$ Raman spectra of eumelanin and/or eumelanin components.

In particular, the Raman peaks at higher wavenumbers (1650-1800 $\left.\mathrm{cm}^{-1}\right)$ are related to $\mathrm{C}=\mathrm{O}$ stretching vibrational modes. Consequently, the observed shoulder at $1663 \mathrm{~cm}^{-1}$ in Fig. (6) can be assigned to $\mathrm{C}=\mathrm{O}$ stretching in quinone structure. Such peak is reported at $1660 \mathrm{~cm}^{-1}$ in Raman spectrum of ortho-quinone, whose structure is similar to that of the DHI unit [37]. In addition, the spectral position of such a shoulder is close to that of the peaks at $1667 \mathrm{~cm}^{-1}$ and $1650 \mathrm{~cm}^{-1}$ of the Raman spectrum of IQ and SQ units, respectively, predicted by Powell et al.. On the contrary, the observed peaks at 1703, 1735 and $1775 \mathrm{~cm}^{-1}$ do not correspond to any vibrational mode neither predicted [15, 16] nor observed [16] inside functional groups of DHI, IQ and SQ structure. Such peaks might be related to $\mathrm{C}=\mathrm{O}$ stretching vibration mode characteristic of carboxylic acid $\mathrm{COOH}$ of DHICA [16] and carboxylic derivatives of pyrrole [10]. In fact, a Raman peak has been predicted at $1743 \mathrm{~cm}^{-1}$ and observed at about $1700 \mathrm{~cm}^{-1}$ in the Raman spectrum of DHICA [16]. Hence, SERS peaks in this spectral range confirm the presence of several carboxylic acid groups in synthetic eumelanin structure.

At lower wavenumbers with respect to those characteristic of $\mathrm{C}=\mathrm{O}$ stretching modes, several peaks related to $\mathrm{C}=\mathrm{C}$ skeletal in-plane vibrations are expected in the 1380 $1625 \mathrm{~cm}^{-1}$ spectral region $[20,37,39-40]$, due to the presence of the two fused benzene and pyrrole rings which form the indole compound. Although these vibrational modes produce only two broad single bands in the deconvoluted Raman spectrum in Fig. (5), centred at 1617 and $1528 \mathrm{~cm}^{-1}$ for aromatic and pyrrole rings, respectively, some of them are clearly resolved in the SERS spectrum of Fig. (6). In particular, the four features at about 1621, 1587, 1545 and $1510 \mathrm{~cm}^{-1}$ are due to $\mathrm{C}=\mathrm{C}$ aromatic ring vibration of indole structure. Indeed, their wavenumbers are in good agreement with Raman features both calculated $[15,16]$ and measured [16, 20] for indole-based compounds. In fact, Powell et al. predicted strong Raman peaks centred at $1640.5,1623.9,1575.3,1533.9 \mathrm{~cm}^{-1}$ for IQ, centred at $1648.9,1585.3,1537.7,1499.3 \mathrm{~cm}^{-1}$ for SQ and centred at 1628.4, $1511.3 \mathrm{~cm}^{-1}$ for DHI [15]. Further, S.P. Nighswander-Rempel et al. calculated feratures centred at 1626.7, 1585.9, $1523.0 \mathrm{~cm}^{-1}$ and they experimentally observed peaks at about $1640,1590,1520 \mathrm{~cm}^{-1}$ in the Raman spectrum of indole unit. [16]. In addition, also S.D. Dieng et al. compared calculated and experimental Raman peaks of indole in solution, finding a blue-shift of few $\mathrm{cm}^{-1}$ for the experimental Raman peaks (1627 and $1586 \mathrm{~cm}^{-1}$ ) with respect to the calculated ones (1618 and $1579 \mathrm{~cm}^{-1}$ ) [20]. Moreover, ring stretching modes of pyrrole might contribute to the peak at $1510 \mathrm{~cm}^{-1}$ in Fig. (6); in particular, one of such pyrrole modes is reported in the $1507-1528 \mathrm{~cm}^{-1}$ spectral range $[16,20,35,36]$. In fact, other spectral features at lower wavenumbers than those due to $\mathrm{C}=\mathrm{C}$ aromatic ring vibrations can be attributed to the vibration modes of pyrrole ring. In particular, pyrrole ring vibrations are reported at 1468,1418 and $1379 \mathrm{~cm}^{-1}$ in [35] as well as they have been observed at 1459 and $1424 \mathrm{~cm}^{-1}$ in [20]. Therefore, the peaks 
at 1469,1422 and $1389 \mathrm{~cm}^{-1}$ in Fig. (6) can be related to $\mathrm{C}=\mathrm{C}$ and $\mathrm{C}=\mathrm{N}$ in-plane vibration of the pyrrole ring.

The SERS peak at $1345 \mathrm{~cm}^{-1}$ in Fig. (6) can be attributed to the C-N stretching vibrational mode, as suggested in [15], or related to a vibrational mode of indole ring, which is reported at $1339 \mathrm{~cm}^{-1}$ in [20].

The spectral range including the SERS peaks at 1303 and $1258 \mathrm{~cm}^{-1}$ (Fig. 6) is mainly related to the vibrational modes characteristic of the carboxylic acid. In particular, the peak at $1303 \mathrm{~cm}^{-1}$ can be related to the $\mathrm{OH}$ deformation mode of $\mathrm{COOH}$ group which is reported in $1380-1280 \mathrm{~cm}^{-1}$ spectral range [37]. Such attribution is confirmed by the fact that no vibrational feature has been predicted neither experimentally observed close to $1300 \mathrm{~cm}^{-1}$ for the indole structure [16]. The peak at $1258 \mathrm{~cm}^{-1}$ can be related to the CO stretching band of carboxylic acid, which is reported in the $1283-1268 \mathrm{~cm}^{-1}$ range [37] and 1315-1200 $\mathrm{cm}^{-1}$ range [40]. A contribution of the $\mathrm{CC}$ and $\mathrm{CN}$ stretching mode of the pyrrole structure is also possible for the peak at $1258 \mathrm{~cm}^{-1}$, as calculated by Ten et al. [36].

The SERS features in the $1250-1000 \mathrm{~cm}^{-1}$ spectral range are related to $\mathrm{CH}$ and $\mathrm{NH}$ in-plane deformation vibrations. In fact, seven Raman peaks calculated and observed by S.D. Dieng et al. in such spectral range for indole compound [20], have been assigned at vibrational modes of both $\mathrm{CH}$ and $\mathrm{NH}$ in-plane deformation. In addition, four $\mathrm{CH}$ in-plane deformation modes are reported at 1237, 1076, 1045 and $1015 \mathrm{~cm}^{-1}$ for the pyrrole structure [35], whereas six $\mathrm{CH}$ in plane deformation modes are reported for the benzene aromatic compounds in the 990-1250 spectral range [37]. Consequently, the SERS peaks at 1213, 1170, 1123, 1075 and $1027 \mathrm{~cm}^{-1}$ in Fig. (6) can be assigned at $\mathrm{CH}$ and $\mathrm{NH}$ inplane deformation modes.

On the lower wavenumber side of the SERS spectrum, the weak feature at $927 \mathrm{~cm}^{-1}$ in Fig. (6) is due to the $\mathrm{CH}$ and $\mathrm{OH}$ out of plane deformation modes of benzene ring, which are reported at $937 \mathrm{~cm}^{-1}$ [36] and $935 \mathrm{~cm}-1$ [39], respectively.

\section{CONCLUSIONS}

Raman and SERS spectra of synthetic eumelanin samples prepared from L-DOPA have been measured, in order to reveal the presence of functional groups related to specific monomeric units of eumelanin. The SERS spectrum, more resolved with respect to the standard Raman one, is characterized by a large number of vibrational modes, which are related to specific functional groups of the eumelanin components. The spectral position of the SERS peaks are consistent with those predicted and observed for the basic eumelanin components and closely-related compounds. In fact, the detailed vibrational features present in the SERS spectrum confirm the presence of DHI, IQ and SQ monomers in synthetic eumelanin, as well as the presence of carboxylic acid and residual components from the synthesis process. The similarity in peak energies between those of the biopolymer and monomers supports the statement that eumelanin consists of a wide ensemble of distinct oligomers.

On the whole, SERS spectra of eumelanin can offer the spectral resolution to resolve and reveal the presence of single functional groups, related to specific eumelanin components. The SERS investigation about synthetic eumelanin (not previously reported in literature) can be the starting point to investigate the vibrational properties of all types of natural eumelanins, inside which the residual protein coat might eventually influence the vibrational spectrum and cause a degree of uncertainty in the attribution of SERS features to functional groups characteristic of the biopolymer.

\section{CONFLICT OF INTEREST}

The authors confirm that this article content has no conflict of interest.

\section{ACKNOWLEDGEMENTS}

We are grateful to D. Fiocco for technical cooperation to the preparation of synthetic eumelanin solutions.

\section{REFERENCES}

[1] Land EJ, Ramsden CA, Riley PA. Tyrosinase autoactivation and the chemistry of ortho-quinone amines. Acc Chem Res 2003; 36: 300-8.

[2] Meredith P, Sarna T. The physical and chemical properties of eumelanin. Pigment Cell Res 2006; 19: 572-94.

[3] Meredith P, Powell BJ, Riesz J, Nighswander-Rempel SP, Pederson MR, Moore EG. Towards structure-property-function relationships for eumelanin. Soft Matter 2006; 2: 37-44.

[4] Nofsinger JB, Weinert EE, Simon JD. Establishing Structure/ Function Relationships for Eulmelanin. Biopolymers 2002; 67: 302-5

[5] Stark KB, Gallas JM, Zajac GW, Eisner M, Golab JT. Spectroscopic Study and Simulation from Recent Structural Models for Eumelanin: II. Oligomers. J Phys Chem B 2003; 107: 11558-62.

[6] Monster AB, Powell BJ, Gentle IR, Meredith P. On the origin of electrical conductivity in the bio-electronic material melanin. Appl Phys Lett 2012; 100: 093701.

[7] Jastrzebska M, Kocot A, Tajber L. Photoconductivity of synthetic dopa-melanin polymer. J Photochem Photobiol B 2002; 66: 201-6.

[8] Morresi L, Ficcadenti M, Pinto N, et al. Optical and electrical behavior of synthetic melanin thin films spray-coated. Energy Procedia 2010; 2: 177-82.

[9] Ambrico M, Cardone A, Ligonzo T, et al. Hysteresis-type currentvoltage characteristics in Au/eumelanin/ITO/glass structure: Towards melanin based memory devices. Org Electr 2010; 11: 1809-14.

[10] Centeno SA, Shamir J. Surface enhanced Raman scattering (SERS) and FTIR characterization of the sepia melanin pigment used in works of art. J Mol Struct 2008; 873: 149-59.

[11] Watt AAR, Bothma JP, Meredith P. The supramolecular structure of melanin. Soft Matter 2009; 5: 3754-60.

[12] Linh Tran M, Powell BJ, Meredith P. Chemical and Structural Disorder in Eumelanins: A Possible Explanation for Broadband Absorbance. Biophys J 2006; 90: 743-52.

[13] Perna G, Frassanito MC, Palazzo G, et al. Fluorescence spectroscopy of synthetic melanin solution. J Lumin 2009; 129: 449.

[14] Perna G, Palazzo G, Mallardi A, Capozzi V. Fluorescence properties of natural eumelanin biopolymer. J Lumin 2011; 131: 1584-8.

[15] Powell BJ, Baruah T, Bernstein N, et al. A First Principles DensityFunctional Calculation of the Electronic and Vibrational Structure of the Key Melanin Monomers. J Chem Phys 2004; 120: 8608-15.

[16] Nighswander-Rempel SP, Olsen S, Mahadevan IB, et al. Effect of dimerization on vibrational spectra of eumelanin precursors. Photochem Photobiol 2008; 84: 613-9.

[17] Huang Z, Lui H, Chen XK, Alajlan A, McLean DI, Zeng H. Raman spectroscopy of in vivo cutaneous melanin. J Biomed Opt 2004; 9: 1198-205.

[18] Capozzi V, Perna G, Gallone A, et al. Raman and optical spectroscopy of eumelanin films. J Mol Struct 2005; 744-747: 71721. 
[19] Sangaletti L, Pagliara S, Vilmercati P, et al. Electronic excitations in synthetic eumelanin aggregates probed by soft X-ray spectroscopies. J Phys Chem B 2007; 111: 5372-6.

[20] Dieng SD, Schelvis JPM. Analysis of measured and calculated Raman spectra of Indole, 3-Methylindole, and Tryptophan on the basis of observed and predicted isotope shifts. J Phys Chem A 2010; 114: 10897-905.

[21] Moskovits M. Surface Enhanced Spectroscopy. Rev Mod Phys 1985; 57: 783-826.

[22] Kneipp K, Kneipp H, Itzkan I, Dasari RR, Feld MS. Ultrasensitive chemical analysis by Raman spectroscopy. Chem Rev 1999; 99: 2957-76.

[23] Kneipp K, Kneipp H, Itzkan I, Dasari RR, Feld MS. Surface enhanced Raman scattering and biophysics. Eur Phys J B 2002; 14: R597-624.

[24] Birembaut F, Perney N, Pechstedt K, Bartlett PN, Russell AE, Baumberg JJ. Sharp-Cornered Liquid Drops by Wetting of Nanoscale Features. Small 2008; 4: 2140-2.

[25] Perney NMB, Baumberg JJ, Zoorob ME, Charlton MDB, Mahnkopf S, Netti CM. Tuning localized plasmons in nanostructured substrates for surface-enhanced Raman scattering. Opt Express 2006; 14: 847-57.

[26] Application notes from Renishaw Diagnostic, RD/Klarite/009, Issue 1.0, May 2010.

[27] Nardou E, Vouagner D, Jurdyc AM, et al. Distance dependence of the Surface Enhanced Raman Scattering effect observed in amorphous $\mathrm{TiO}_{2}$ on nanostructured gold. Opt Mater 2011; 33: 1907-10.

[28] Radzol ARM, Lee YK, Mansor W, Yahaya SR. Proceedings - 2012 IEEE 8th International Colloquium on Signal Processing and Its Applications, CSPA 2012; art. no. 6194781: 2012; pp. 505-9.

[29] Alexander TA, Le DM. Characterization of a commercialized SERS-active substrate and its application to the identification of intact Bacillus endospores. Appl Opt 2007; 46: 3878-90.

[30] Martin-Sanchez PM, Sanchez-Cortez S, Lopez-Tobar E, et al. The nature of black stains in Lauscaux Cave, France, as revealed by surface enhanced Raman spectroscopy. J Raman Spectrosc 2012; 43: 464-7.

[31] Zeise L. Analytical methods for characterization and identification of eumelanins. In: Zeise L, Chedekel MR, Fitzpatrick TB, Eds. Melanin: its role in human photoprotection. Overland Park, KS: Valdenmar Publishing 1995; pp. 65-79.

[32] Bridelli MG. Self-assembly of melanin studied by laser light scattering. Biophys Chem 1998; 73: 227-39.

[33] Kruk I, Lichszteld K, Bounias M, Kadna A, Kubera-Nowakowska L. Formation of active oxygen species during autoxidation of DOPA. Chemosphere 1999; 39: 443-53.

[34] Abbas M, Ali M, Sha SK, et al. Control of structural, electronic and optical properties of eumelanin films by electrospray deposition. J Phys Chem B 2011; 115: 11199-207.

[35] Jones RA. In: Katritzky AR, Boulton AJ, Eds. Advances in heterocyclic chemistry, physicochemical properties of pyrroles. New York: Academic Press 1970; vol. 11: p. 443.

[36] Ten GN, Yakovleva AA, Burova TG, Berezin VI, Baranov VI. Modeling vibrational spectra of indole in water. J Appl Spectrosc 2010; 77: 502-9.

[37] Socrates G. Infrared and raman characteristic group frequencies. Tables and charts. New York: John Wiley \& Sons 1994.

[38] Dollish FR, Fateley WG, Bentley FF. Characteristic raman frequencies of organic compounds. New York: John Wiley \& Sons 1974.

[39] Bellamy LJ. The infrared spectra of complex molecules. New York: John Wiley \& Sons 1962.

[40] Lin-Vien D, Colthup NB, Fateley WG, Grasselli JG. The handbook of infrared and raman characteristic frequencies of organic molecules. New York: Academic Press 1991.

[41] Hyogo R, Nakamura A, Okuda H, Wakamatsu K, Ito S, Sota T. Mid-infrared vibrational spectroscopic characterization of 5,6dihydroxyindole and eumelanin derived from it. Chem Phys Lett 201; 517: 211-6. 